\title{
Aprendizaje-Servicio y rendimiento académico del alumnado universitario
}

\author{
Service-Learning and academic achievement of university students
}

\author{
Ígor Mella-Núñez*, Miguel Ángel Santos-Rego*, Xosé Manuel Malheiro-Gutiérrez** \\ *Grupo de Investigación ESCULCA / Rede de Investigación RIES - Universidade de Santiago de Compostela, **Grupo de \\ Investigación ESCULCA - Universidade da Coruña
}

\begin{abstract}
Resumen
El objetivo de este trabajo es conocer la relación existente entre la implementación de programas de Aprendizaje-Servicio (ApS) en la universidad y el rendimiento académico del alumnado. Para ello, partiendo de que el ApS es una práctica pedagógica potencialmente efectiva desde un punto de vista académico, realizamos un análisis bibliográfico a fin de conocer el impacto que esta metodología tiene en el logro académico del alumnado. Esta revisión tendrá alcance internacional debido al escaso recorrido que tal acento ha tenido en nuestro país, donde una amplia parte de los estudios se han centrado, especialmente, en la dimensión cívico-social.

Palabras clave: aprendizaje-servicio, rendimiento académico, educación superior, innovación educativa.
\end{abstract}

\begin{abstract}
This paper is aimed at finding the relationship between the implementation of Service-Learning programs at the university level, and students' academic performance. To this end, first, service-learning should be understood as an effective teaching practice from an academic point of view, and a literature review should be carried out to understand the impact that this methodology has on students' academic performance. Thus, an international literature review is performed, due to the limited scope that this approach had so far in our country, where most research studies focused particularly on the civic and social dimension.
\end{abstract}

Keywords: service-learning, academic achievement, higher education, educational innovation.

\section{Introducción}

Hablar de rendimiento académico es hacerlo de una de las variables que mayor interés concita en la investigación educativa. Todo proceso formativo se orienta, en mayor o menor medida, a alcanzar el éxito, como resultado de combinar esfuerzo y habilidad en los alumnos, a lo que hemos de añadir el modo en que influye el contexto (Edel, 2003).

En la universidad, la atención prestada al rendimiento académico se explica apelando, como señalan Martín y González Afonso (2010), al firme desarrollo de una cultura de evaluación de la calidad. En este sentido, el logro académico del alumnado se considera un indicador fiable de la calidad en los procesos formativos que allí tienen lugar, pues muestra grados de eficacia y eficiencia institucional.
Si entendemos, pues, el rendimiento académico como un reflejo de la eficacia universitaria, esto es, el modo en que se cumplen objetivos al respecto, no puede extrañar que su mejora sea objeto de atención en todas las agendas de política y legislación educativas. En nuestro país, la Ley Orgánica 6/2001 de Universidades, junto con la declaración de Bolonia, en el marco comunitario, buscan fehacientemente la mejora en la calidad y competitividad del sistema universitario. Lo cual exige la mejora previa de los procesos formativos.

De otra parte, las reformas universitarias que estamos viviendo en la actualidad se fundamentan en un aprendizaje centrado en el alumno, potenciando así su autonomía e implicación (Duran y Flores, 2014), confiando en que la implementación de metodologías pedagógicas innovadoras conlleve una mejora integral de la educación superior, lo que habría de implicar una mejora en el aprendizaje del alumnado y/o en su rendimiento académico.

No obstante, y a pesar del papel que a lo largo de la historia han desempeñado las universidades como alma mater de las sociedades, actualmente se enfrentan a un nuevo desafío: el incremento del número de alumnos. En esta coyuntura, de mayor conexión con la sociedad civil, es preciso cuidar el rendimiento académico de ese creciente número de alumnos, a todas luces muy heterogéneos entre sí.

En España, según datos del Instituto Nacional de Estadística (2015), había una población en 2014 de 46.512.199 personas. En el curso académico 2013-2014 el número de alumnos matriculados en enseñanzas universitarias de grado y master era de 1.532 .728 , lo que supone una tasa de escolarización en educación universitaria del 29,7\% para el grupo de edad 18-24 años (Ministerio de Educación, Cultura y Deporte, 2015). Tal masificación ha traído consigo un incremento del fracaso académico debido a la dificultad de adaptar un modelo de universidad obsoleto, orientado según criterios elitistas, a las exigencias de una universidad más moderna y democrática (Rodríguez Espinar, Fita, y Torrado, 2003; Tejedor y García-Valcárcel, 2007).

Debido a este contexto de masificación y expansión universitaria, las metodologías tradicionales pueden resultar poco eficaces ante un alumnado tan amplio y diverso. Al contrario, es necesario implementar aprendizajes basados en prácticas pedagógicas que fomenten un aprendizaje más cercano a la experiencia

Este trabajo se deriva de un primer análisis bibliográfico realizado dentro del Proyecto de I+D+i “Aprendizaje-Servicio e Innovación en la Universidad. Un programa para la mejora del rendimiento académico y el capital social de los estudiantes”, financiado por el Ministerio de Economía y Competitividad como proyecto de $\mathrm{I}+\mathrm{D}+\mathrm{i}$ "Retos Investigación" del Programa Estatal de I+D $+\mathrm{i}$ Orientada a los Retos de la Sociedad (EDU2013-41687-R)

Correspondencia: Ígor Mella Núñez, igor.mella@usc.es

Selección y peer-review bajo responsabilidad del Grupo de Investigación G000422-GIPDAE, Universidade da Coruña, España. 
del alumno, pues puede que permitan transmitir las ideas abstractas de la universidad de un modo más eficaz a los estudiantes, ya que la combinación entre experiencia y estudio permitirá comprender lo trabajado en clase mediante la práctica, aspecto que tendrá consecuencias notorias en la optimización y profundidad del aprendizaje adquirido por el alumnado (Kolb, 1984).

Entre las metodologías innovadoras que buscan mejorar continuamente los procesos de enseñanza-aprendizaje, se encuentra el Aprendizaje-Servicio (ApS), metodología heredera de los principios pedagógicos de John Dewey, artífice intelectual del denominado pragmatismo americano, corriente en la que se acentúa el poder educativo asociado a la experiencia de los alumnos en un marco comunitario, esperando que saquen ventaja tanto el alumnado como el mismo contexto social (Santos Rego y Lorenzo Moledo, 2009; Santos Rego, 2013).

Podemos definir el ApS, por tanto, como "una propuesta educativa que combina procesos de aprendizaje y de servicio a la comunidad en un solo proyecto bien articulado en el que los participantes se forman al trabajar sobre necesidades reales del entorno con el objetivo de mejorarlo" (Puig, Batlle, Bosch, y Palos, 2007, p. 20). La relación entre el currículo y el servicio permite al alumnado universitario mejorar su conocimiento de una disciplina y ampliar su sentido de la responsabilidad social, por lo que el Aprendizaje-Servicio se configura como una metodología educativa innovadora que se asocia a patrones de mejora cognitiva y social.

Así, representamos el ApS a través de la dualidad que, ciertamente, desliza su propia denominación: aprendizaje, en tanto que dimensión propia del rendimiento académico de los estudiantes; y servicio, en tanto que dimensión singularmente vinculable a su compromiso cívico-social (Santos Rego, Sotelino, y Lorenzo Moledo, 2015). Uno de los retos de este enfoque, a juzgar por la literatura científica, tiene que ver con las posibles vías de equilibración entre dichas dimensiones, pues no debemos olvidar que hasta el momento la hegemonía investigadora parece favorecer a los factores del servicio, en detrimento de los más directamente relacionados con el aprendizaje y el rendimiento académico del alumnado (Strage, 2000; Hart y King, 2007).

La excelencia universitaria será insuficiente si no atiende también al desarrollo de competencias de carácter cívico y social (Santos Rego y Lorenzo Moledo, 2007). Por ello, creemos que existe una necesidad de estudiar las posibles relaciones que se puedan establecer entre la implementación de programas de Aprendizaje-Servicio y el rendimiento del alumnado, ya que el logro académico se convierte en uno de los fines más valorados y perseguidos, especialmente en la enseñanza superior.

No obstante, para establecer la relación entre ApS y rendimiento, es necesario tener en cuenta que ante un cambio en los esquemas tradicionales del proceso de enseñanza-aprendizaje, se requiere un cambio en los sistemas de evaluación. Por tanto, ante la introducción de metodologías innovadoras como el Aprendizaje-Servicio en la universidad, se requiere una transformación de los sistemas de evaluación tradicionales, centrados en medir competencias de orden inferior, como la memoria o la comprensión, en detrimento de competencias de orden superior, como el análisis, la síntesis o el pensamiento crítico (Ives y Obenchain, 2006).

Así, la pregunta clave podría ser: ¿influye la implementación de programas de Aprendizaje-Servicio en el rendimiento académico del alumnado universitario? La bibliografía, especialmente en el ámbito nacional, es escasa, pues en la mayoría de los trabajos de investigación existentes los efectos del ApS en el alumnado universitario se estudian desde la dimensión del componente cívico.

Para arrojar luz sobre el tema, el objetivo principal de este estudio no es otro que el de realizar una revisión bibliográfica, de carácter internacional, acerca de las posibles implicaciones que el Aprendizaje-Servicio puede tener en el rendimiento de los estudiantes en enseñanzas universitarias. Con los siguientes objetivos específicos:

- Analizar el modo en que se produce el impacto del ApS en el logro académico.

- Descubrir los motivos que propician el impacto de los programas de ApS en el rendimiento.

- Determinar aquellos aspectos o dimensiones del rendimiento académico en los que pueden tener efectos las prácticas de ApS.

\section{Método}

En el estudio se siguió una metodología de investigación documental, de modo que fuese posible conocer el estado de la cuestión a través de la recopilación, selección y análisis de trabajos previos. Para ello se accedió tanto a obras de carácter nacional como internacional, empleando bases de datos como Dialnet para trabajos nacionales, o ERIC para trabajos internacionales. Con este procedimiento se pudo constatar la escasez de estudios sobre la cuestión en el contexto español, por lo que se prestó mayor atención a la obtención y análisis de trabajos de los Estados Unidos de América, donde la investigación sobre ApS - amén de su institucionalización en la educación superior-, se encuentra en un estado de mayor desarrollo.

Así, centramos nuestra búsqueda en trabajos que analizasen el impacto que, en términos académicos, tiene el Aprendizaje-Servicio sobre los estudiantes universitarios. Para ello, se privilegiaron los estudios de carácter experimental, en los que, a través de comparaciones entre diferentes grupos de alumnos, fuera posible establecer los efectos de la implementación de uno o varios programas sobre ciertas dimensiones o indicadores del rendimiento académico.

\section{Resultados}

Permítasenos, en primer lugar, presentar datos de rendimiento académico en el sistema universitario español. Esos datos fueron dados a conocer por el Ministerio de Educación, Cultura y Deporte (2015), si bien están referidos al curso 2012-2013. Pues bien, la tasa de rendimiento, entendida como la relación 
porcentual entre el número de créditos superados y el número de créditos matriculados, se situaba en un $76,3 \%$ para estudios de grado; y en un $71 \%$ para estudios de primer y segundo ciclo; lo cual pone de manifiesto que entre un $25 \%$ y un $30 \%$ de los créditos matriculados no son superados por los alumnos, respectivamente.

Los datos señalados suscitan una razonable preocupación por los niveles de fracaso académico existentes en la educación superior. Lo que urge, por ello, son nuevos enfoques pedagógicos a fin de intentar dinámicas renovadoras en el proceso de enseñanza-aprendizaje. Una de las que se tienen apuntado es, justamente, el ApS. Porque, en palabras de Palos (2009), se trata de facilitar el aprendizaje y el desarrollo integral del alumnado, sin dejar de favorecer los buenos resultados académicos.

Partimos, pues, de la pertinencia del ApS en la enseñanza universitaria y lo asumimos como un potencial revulsivo ante los niveles de fracaso existentes. Así, confiamos en que el impacto de los programas de Aprendizaje-Servicio en el logro académico no se entienda de un modo aislado, sino interconectado con un conjunto mayor de dimensiones, o como resultado del impacto o efecto en dichas dimensiones.

Con esta idea de fondo, presentaremos ahora diversas clasificaciones de las dimensiones en las que pueden producirse determinados efectos a partir de la implementación de programas de ApS. Para comenzar, en un meta-análisis realizado en los Estados Unidos, en el que se analizan 62 programas de ApS con 11.837 estudiantes, de los cuales un $68 \%$ eran universitarios, se derivó que el Aprendizaje-Servicio muestra efectos positivos en cinco áreas: actitud hacia uno mismo, actitudes hacia la universidad y el aprendizaje, el compromiso cívico, las competencias sociales y el rendimiento académico (Celio, Durlak, y Dymnicki, 2011). Los datos extraídos de este meta-análisis hacen que nos situemos en la posición, defendida también por Simonet (2008), de que para conocer el impacto de los programas de ApS en el rendimiento del alumnado, conviene verlo como una relación indirecta, a través de ciertas variables mediadoras, entre las cuales estaría el incremento en las relaciones con el profesorado, una mayor participación en las actividades del campus, una mayor satisfacción con el ambiente del campus, o aún el incremento en el aprendizaje activo del alumno.

En definitiva, el soporte teórico podría venir dado por el modelo de Furco (2005), para el que, por más que la perspectiva tradicional defienda un vínculo directo entre el ApS y el rendimiento académico del alumnado universitario, en realidad ese efecto se produciría a través de una serie de factores mediadores: autoestima, empoderamiento, conducta prosocial, motivación y compromiso. Por tanto, es erróneo -viene a decirseestablecer un relación directa entre las experiencias de ApS y los logros académicos de los estudiantes, pues las mejoras en el rendimiento y en el aprendizaje se conseguirán focalizando el efecto de estas prácticas pedagógicas en las variables mediadoras anteriormente citadas.

Así las cosas, y después de haber conocido el modo en que se produce el impacto de las prácticas de
Aprendizaje-Servicio en el rendimiento, abordaremos los motivos que están detrás de esa relación positiva. Para empezar, Scales, Roehlkepartain, Neal, Kielsmeier, y Benson (2006), en un estudio con 1.799 centros no universitarios, presentan una serie de motivos que, en su opinión, hacen que el Aprendizaje-Servicio influya, incluso de un modo académicamente decisivo, en el alumnado. En primer lugar, defienden una mejora en el compromiso de los estudiantes y en su visión de la institución educativa, al verse envueltos en el currículo y en la construcción del conocimiento. En segundo lugar, destacan la importancia de esta metodología en el rendimiento en tanto que llega a transmitir a los estudiantes un sentimiento de efectividad, permitiéndoles visibilizar la utilidad de los conocimientos adquiridos. De un modo similar, Hart y King (2007), en un estudio con 63 profesores en formación, de los cuales 34 participaron en un programa de ApS, sostienen que el impacto de esta práctica pedagógica en el aprendizaje y rendimiento del alumnado se debe al modo en que favorece su responsabilidad, haciéndolos dueños de su aprendizaje y dotándolos de voz propia.

Sin embargo, a pesar de los motivos apuntados, Astin y Sax (1998) afirman que existe una reticencia más o menos extendida a la participación de los alumnos en actividades de servicio a la comunidad, por exigirles un tiempo que podrían dedicar a actividades y tareas exclusivamente académicas. No obstante, estos mismos autores, en un estudio con 42 instituciones universitarias y 3.450 estudiantes, descubrieron que las actividades al servicio de la comunidad contribuían a producir efectos positivos en diez dimensiones estrictamente relacionadas con el rendimiento académico del alumnado: la nota media, la persistencia en los estudios, las aspiraciones académicas, el incremento de conocimientos generales, el incremento de conocimientos disciplinares, la preparación para los siguientes niveles, el autoconcepto académico, el tiempo dedicado a estudiar, el trabajo extra hecho para las asignaturas, y el contacto y relación con el profesorado.

Por último, nos centraremos en conocer el posible impacto que los programas universitarios de ApS pueden tener en tres dimensiones directamente relacionadas con el rendimiento del alumnado y que, además de ser las más empleadas por los autores, son un claro reflejo del éxito o fracaso académico: las tasas de abandono, los índices de graduación y la nota media.

En primer lugar, en relación a las tasas de abandono del alumnado en educación superior, Smink y Reimer (2005), en un informe del National Dropout Prevention Center, proponen el Aprendizaje-Servicio, al lado de otras catorce medidas, como una metodología recomendable en cualquier tentativa de reducir las tasas de abandono en la universidad. Por su parte, Gallini y Moely (2003), en un trabajo de investigación con 313 estudiantes universitarios, de los que 142 habían participado en programas de $\mathrm{ApS}$, concluían que los alumnos participantes en este tipo de programas muestran un mayor índice de persistencia en la universidad. Y destacan que son los aspectos académicos de la metodología en cuestión, caso de su relación con el 
currículo de la materia, los que tienen un mayor efecto en la retención del alumnado.

A propósito de la persistencia del alumnado, puede que, como consecuencia, tenga algo que ver con la tasa de graduación. Sobre esta dimensión, Lockeman y Pelco (2013) confirmaron que, después de estudiar ex post facto durante seis años a un número de 3.458 alumnos de una universidad pública estadounidense, quienes habían participado en programas de Aprendizaje-Servicio mostraban una tasa de graduación notablemente mayor, referida a un período de seis años (73\% frente a $48 \%)$. En un estudio similar en la California State University (Campus de Fresno), resultó que aquellos alumnos de primer año que participaban en programas de ApS tenían una mayor probabilidad de terminar los estudios en un período de cuatro o cinco años, a diferencia de aquellos que no participaban en los mismos (Leimer, Yue, y Rogulkin, 2009). Con estos datos, podría decirse, desde luego, que en los Estados Unidos de América el incremento del número de alumnos que participan en programas de ApS se ha convertido, al menos parcialmente, en objetivo institucional, dado el interés por aumentar las tasas de graduación en la universidad (Lockeman y Pelco, 2013).

Por último, poniendo la atención en la nota media como el indicador hegemónico del rendimiento académico en todos los niveles del sistema educativo, especialmente en la universidad, también encontramos estudios evidenciando los efectos positivos del ApS en esa dimensión. Uno de ellos fue el de Strage (2000). El trabajo reunió a 477 alumnos universitarios, de los que 166 participaban en programas de ApS. La conclusión resultó meridiana: aquellos con los que se empleaba la metodología ApS obtenían una mejor nota media en los exámenes finales.

En una línea similar, Astin, Vogelgesang, Ikeda, y Yee (2000) estudiaron a 22.236 estudiantes universitarios. De ellos, un 29,9\% había participado en actividades de ApS, un $46,5 \%$ en actividades de servicio tradicional y un 23,6\% no había participado en ningún tipo de servicio. De nuevo, su conclusión era que el ApS influye en la nota media de un modo directo, sin necesidad de variables intermedias. La diferencia con un tipo de servicio más tradicional era igualmente prístina: en ese servicio tradicional, la influencia del profesor es determinante a la hora de mejorar la nota media del alumnado.

Estos autores (citados en último lugar) proponen dos posibles causas que explicarían el impacto del Aprendizaje-Servicio en la nota media: por un lado, el hecho de que este tipo de actividades requiera menos esfuerzo por parte de los estudiantes, ya que podría resultarles más fácil; y por otro, la posibilidad de que un aumento en el compromiso académico pueda acarrear una posterior mejora en la nota media. Nuestro supuesto preferido es el segundo, toda vez que son numerosos los autores que llegan a considera esta práctica educativa como elemento clave en la mejora del compromiso académico de los estudiantes (Strage, 2000; Gallini y Moely, 2003; Scales et al., 2006; Simonet, 2008). Sería, entonces, tal compromiso el que podría erigirse en factor claramente influyente en una posterior mejora de la nota media $\mathrm{y}$, por ende, del logro académico.

\section{Discusión y Conclusiones}

Todo lo que hemos tenido la oportunidad de revisar y estudiar indica que el efecto de esta metodología se antoja, en general, beneficioso y positivo. No obstante, queremos destacar, en primer lugar, la escasa bibliografía existente sobre la cuestión concreta que hemos abordado. Con el sesgo de tener que limitar los hallazgos presentados a trabajos planteados y desarrollados en los Estados Unidos, cuyo recorrido en este terreno es algo bien conocido. Además, esa escasez bibliográfica podría explicarse en función de una hegemonía investigadora en la dimensión cívico-social del $\mathrm{ApS}$, en detrimento de aquella más relacionada con el aprendizaje y el rendimiento del alumnado.

Aun así, el trabajo efectuado ha facilitado una cierta constatación de que la implementación de programas de Aprendizaje-Servicio muestra efectos positivos en ciertas dimensiones o indicadores del logro académico del alumnado universitario, entre las cuales hemos podido contar las tasas de abandono, de graduación, o el mismo promedio de las cualificaciones.

Se destaca el hecho de que tal efecto no se produce de un modo directo, ya que todo apunta a que la mejora del rendimiento tiene lugar mediante cambios producidos en otras variables mediadoras, caso de la autoestima o el empoderamiento.

En definitiva, convendrá otorgar la importancia debida, desde el paradigma aceptado, a la visualización del impacto que pueda tener la mejora de estas dimensiones mediadoras, sin dejar de reparar en los posibles beneficios académicos para los estudiantes y la calidad de su aprendizaje.

\section{Referencias}

Astin, A. W., y Sax, L. J, (1998). How undergraduates are affected by service participation? Journal of College Student Development, 39(3), 251-263.

Astin, A. W., Vogelgesang, L. J., Ikeda, E. K., y Yee, J. A. (2000). How Service-Learning affects students. Los Angeles: Higher Education Research Institute. Recuperado de http://heri.ucla.edu/pdfs/hslas/hslas.pdf

Celio, C. I., Durlak, J., y Dymnicki, A. (2011). A meta-analysis of the impact of Service-Learning on students. Journal of Experiential Education, 34(2), 164-181.

Duran, D., y Flores, M. (2014). Prácticas de tutoría entre iguales en universidades del Estado español y de Iberoamérica. Revista Iberoamericana sobre Calidad, Eficacia y Cambio en Educación, 13(1), 5-17. Recuperado

de http://www.rinace.net/reice/numeros/arts/vol13nu m1/art1.pdf

Edel, R. (2003). El rendimiento académico: concepto, investigación y desarrollo. Revista Electrónica Iberoamericana sobre Calidad, Eficacia y Cambio en Educación, 1(2). Recuperado de http://www.ice.deusto.es/RINACE/reice/volln2/Ed el.pdf

Furco, A. (2005). Impacto de los proyectos de aprendizaje servicio. Aprendizaje y servicio solidario en la Educación Superior y en los sistemas educativos 
latinoamericanos. Actas del $7^{\circ} \quad$ Seminario Internacional Aprendizaje y Servicio Solidario (pp. 19-26). Buenos Aires: CLAYSS. Recuperado de http://www.clayss.org.ar/seminario/actas/2005_Act as7.pdf

Gallini, S. M., y Moely, B. E. (2003). Service-Learning and engagement, academic challenge, and retention. Michigan Journal of Community Service Learning, 10(1), 5-14.

Hart, S. M., y King, J. R. (2007). Service learning and literacy tutoring: Academic impact on pre-service teachers. Teacher and Teaching Education, 23, 323-338

Instituto Nacional de Estadística (2015). Página web del Instituto Nacional de Estadística. Recuperado de http://www.ine.es/inebaseDYN/cp30321/cp_inicio. htm

Ives, B., y Obenchain, K. (2006). Experiential education in the classroom and academic outcomes: for those who want it all. Journal of Experiential Education, 29(1), 61-77.

Kolb, D. A. (1984). Experiential learning. Experience at the source of learning and development. Nueva Jersey: Prentice Hall.

Leimer, C., Yue, H., y Rogulkin, D. (2009). Does Service-Learning help students succeed? Assessing the effects of Sevice-Learning at California State University-Fresno. Recuperado de http://files.eric.ed.gov/fulltext/ED521013.pdf

Lockeman, K. S., y Pelco, L. E. (2013). The relationship between Service-Learning and degree completion. Michigan Journal of Community Service Learning, 20(1), 18-30.

Martín, E., y González Afonso, M. (2010). El rendimiento académico del alumnado de nuevo ingreso en la Universidad de La Laguna. La Laguna: Servicio de Publicaciones Universidad de La Laguna.

Ministerio de Educación, Cultura y Deporte (2015). Datos básicos del sistema universitario español. Curso 2014-2015. Madrid: Autor.

Palos, J. (2009). ¿Por qué hacer actividades de aprendizaje servicio? En J. M. Puig (Coord.), Aprendizaje servicio (ApS). Educación y compromiso cívico (pp. 151-161). Barcelona: Graó.

Puig, J. M., Batlle, R., Bosch, C., y Palos, J. (2007). Aprendizaje servicio. Educar para la ciudadanía. Madrid: Octaedro.

Rodríguez Espinar, S., Fita, E., y Torrado, M. (2003). El rendimiento académico en la transición secundaria-universidad. Revista de Educación, 334, 391-414.

Santos Rego, M. A. (2013). ¿Para cuándo las universidades en la agenda de una democracia fuerte? Educación, aprendizaje y compromiso cívico en Norteamérica. Revista de Educación, 361, 565-590.

Santos Rego, M. A., y Lorenzo Moledo, M. M. (2007). Universidade e construcción da sociedade civil. Vigo: Xerais.

Santos Rego, M. A., y Lorenzo Moledo, M. M. (2009). Educación para a cidadanía e os profesores. Vigo: Xerais.
Santos Rego, M. A., Sotelino, A., y Lorenzo Moledo, M. M. (2015). Aprendizaje-Servicio y misión cívica de la universidad. Una propuesta de desarrollo. Barcelona: Octaedro (en prensa).

Scales, P. C., Roehlkepartain, E. C., Neal, M., Kielsmeier, J. C., y Benson, P. L. (2006). Reducing academic achievement gaps: The role of Community Service and Service Learning. Journal of Experiential Education, 29(1), 38-60.

Simonet, D. (2008). Service-Learning and academic success: The links to retention research. Recuperado de http://www.compact.org/wp-content/uploads/resou rces/downloads/MN-SL_and_academic_success.pdf

Smink, J., y Reimer, M. S. (2005). Fifteen effective strategies for improving student attendance and truancy prevention. Clemson: National Dropout Prevention Center. Recuperado de: http://www.dpi.state.nd.us/Title1/progress/present/ 15 ways.pdf

Strage, A. A. (2000). Service-Learning: Enhancing student learning outcomes in a college-level lecture course. Michigan Journal of Community Service Learning, 7, 5-13.

Tejedor, F. J., y García-Valcárcel, A. (2007). Causas del bajo rendimiento del estudiante universitario (en opinión de los profesores y alumnos). Propuestas de mejora en el marco del EEES. Revista de Educación, $342,443-473$ 